\title{
Staggered ladder spectra
}

\author{
E. Arvedson, ${ }^{1}$ M. Wilkinson, ${ }^{2}$ B. Mehlig, ${ }^{1}$ and K. Nakamura ${ }^{3}$ \\ ${ }^{1}$ Department of Physics, Göteborg University, 41296 Gothenburg, Sweden \\ ${ }^{2}$ Faculty of Mathematics and Computing, The Open University, \\ Walton Hall, Milton Keynes, MKY 6AA, England \\ ${ }^{3}$ Department of Applied Physics, Osaka City University, Sumiyoshi-ku, Osaka 558-8585, Japan
}

\begin{abstract}
We exactly solve a Fokker-Planck equation by determining its eigenvalues and eigenfunctions: we construct nonlinear second-order differential operators which act as raising and lowering operators, generating ladder spectra for the odd and even parity states. These are staggered: the odd-even separation differs from even-odd. The Fokker-Planck equation describes, in the limit of weak damping, a generalised Ornstein-Uhlenbeck process where the random force depends upon position as well as time. Our exact solution exhibits anomalous diffusion at short times and a stationary non-Maxwellian momentum distribution.
\end{abstract}

PACS numbers: 05.40.-a,05.45.-a,02.50.-r,05.40.Fb

There are only few physically significant systems with ladder spectra (exactly evenly spaced energy levels). Examples are the harmonic oscillator and the Zeemansplitting Hamiltonian. In this letter we introduce a pair of exactly solvable eigenvalue problems. We use them to solve two (closely related) physically significant extensions of a classic problem in the theory of diffusion, namely the Ornstein-Uhlenbeck process. These eigenvalue problems have ladder spectra. Our systems differ from the examples above in that their spectra consist of two ladders which are staggered; the eigenvalues for eigenfunctions of odd and even symmetry do not interleave with equal spacings. We introduce a new type of raising and lowering operators in our solution, which are nonlinear second-order differential operators. Our generalised Ornstein-Uhlenbeck systems exhibit anomalous diffusion at short times, and non-Maxwellian velocity distributions at equilibrium; we obtain exact expressions which are analogous to results obtained for the standard Ornstein-Uhlenbeck process.

1. Ornstein-Uhlenbeck processes. Before we discuss our extension of the Ornstein-Uhlenbeck process, we describe its usual form. This considers a particle of momentum $p$ subjected to a rapidly fluctuating random force $f(t)$ and subject to a drag force $-\gamma p$, so that the equation of motion is $\dot{p}=-\gamma p+f(t)$. The random force has statistics $\langle f(t)\rangle=0,\left\langle f(t) f\left(t^{\prime}\right)\right\rangle=C\left(t-t^{\prime}\right)$ (angular brackets denote ensemble averages throughout). If the correlation time $\tau$ of $f(t)$ is sufficiently short $(\gamma \tau \ll 1)$, the equation of motion may be approximated by a Langevin equation: $\mathrm{d} p=-\gamma p \mathrm{~d} t+\mathrm{d} w$, where the Brownian increment $\mathrm{d} w$ has statistics $\langle\mathrm{d} w\rangle=0$ and $\left\langle\mathrm{d} w^{2}\right\rangle=2 D_{0} \mathrm{~d} t$. The diffusion constant is $D_{0}=\frac{1}{2} \int_{-\infty}^{\infty} \mathrm{d} t\langle f(t) f(0)\rangle$. This problem is discussed in many textbooks (for example [1]); it is easily shown that the variance of the momentum (with the particle starting at rest) is

$$
\left\langle p^{2}(t)\right\rangle=[1-\exp (-2 \gamma t)] D_{0} / \gamma,
$$

that the equilibrium momentum distribution is Gaussian and that the particle (of mass $m$ ) diffuses in space with diffusion constant $\mathcal{D}_{x}=D_{0} / m^{2} \gamma^{2}$.
In many situations the force on the particle will be a function of its position as well as of time. Our extension of the Ornstein-Uhlenbeck process is concerned with what happens in this situation when the damping is weak. We consider a force $f(x, t)$ which has mean value zero, and a correlation function $\left\langle f(x, t) f\left(x^{\prime}, t^{\prime}\right)\right\rangle=$ $C\left(x-x^{\prime}, t-t^{\prime}\right)$. The spatial and temporal correlation scales are $\xi$ and $\tau$ respectively. If the momentum of the particle is large compared to $p_{0}=m \xi / \tau$, then the force experienced by the particle decorrelates more rapidly than the force experienced by a stationary particle. Thus, if the damping $\gamma$ is sufficiently weak that the particle is accelerated to a momentum large compared to $p_{0}$, the diffusion constant characterising fluctuations of momentum will be smaller than $D_{0}$. The impulse of the force on a particle which is initially at $x=0$ in the time from $t=0$ to $t=\Delta t$ is

$$
\Delta w=\int_{0}^{\Delta t} \mathrm{~d} t f(p t / m, t)+O\left(\Delta t^{2}\right)
$$

If $\Delta t$ is large compared to $\tau$ but small compared to $1 / \gamma$, we can estimate $\left\langle\Delta w^{2}\right\rangle=2 D(p) \Delta t$, where

$$
D(p)=\frac{1}{2} \int_{-\infty}^{\infty} \mathrm{d} t C(p t / m, t) .
$$

When $p \ll p_{0}$ we recover $D(p)=D_{0}$. When $p \gg p_{0}$, we can approximate (3) to obtain

$$
D(p)=\frac{D_{1} p_{0}}{|p|}+O\left(p^{-2}\right), D_{1}=\frac{m}{2 p_{0}} \int_{-\infty}^{\infty} \mathrm{d} X C(X, 0) .
$$

If the force is the gradient of a potential, $f(x, t)=$ $\partial V(x, t) / \partial x$, then $D_{1}=0$. In this case, expanding the correlation function in (3) in its second argument gives $D(p) \sim D_{3} p_{0}^{3} /\left|p^{3}\right|$, where $D_{3}$ may be expressed as an integral over the correlation function of $V(x, t)$. To summarize: the momentum diffusion constant is a decreasing function of momentum, such that $D(p) \sim|p|^{-1}$ for a generic random force, or $D(p) \sim|p|^{-3}$ for a gradient force. 
2. Fokker-Planck equation. The probability density for the momentum, $P(p, t)$, satisfies a Fokker-Planck (generalised diffusion) equation. Following arguments in [1], one obtains

$$
\partial_{t} P=\partial_{p}\left(\gamma p P+D(p) \partial_{p} P\right) .
$$

Sturrock [2] introduced a related Fokker-Planck equation (without the damping term) and also gave an expression for $D(p)$ analogous to equation (3). His work was applied to the stochastic acceleration of particles in plasmas, and subsequent contributions have concentrated on refining models for the calculation of $D(p)$ (see, for example, $\underline{3}$, [4]). In the following we obtain exact solutions to (5) in the cases where $D(p)=D_{1} p_{0} /|p|$ (which we consider first) and $D(p)=D_{3} p_{0}^{3} /|p|^{3}$ (which can be treated in the same way, and for which we discuss the results at the end of the paper).

Introducing dimensionless variables $\left(t^{\prime}=\gamma t\right.$ and $z=$ $\left.p\left(\gamma / p_{0} D_{1}\right)^{1 / 3}\right)$, the Fokker-Planck equation for the case where $D(p) \propto 1 / p$ becomes

$$
\partial_{t^{\prime}} P=\partial_{z}\left(z P+|z|^{-1} \partial_{z} P\right) \equiv \hat{F} P .
$$

It is convenient to transform the Fokker-Planck operator $\hat{F}$ to a Hermitian form, which we shall refer to as the Hamiltonian operator

$$
\hat{H}=P_{0}^{-1 / 2} \hat{F} P_{0}^{1 / 2}=\frac{1}{2}-\frac{|z|^{3}}{4}+\frac{\partial}{\partial z} \frac{1}{|z|} \frac{\partial}{\partial z}
$$

where $P_{0}(z) \propto \exp \left(-|z|^{3} / 3\right)$ is the stationary solution satisfying $\hat{F} P_{0}=0$. We solve the diffusion problem by constructing the eigenfunctions of the Hamiltonian operator. In the following we make free use of the Dirac notation [5] of quantum mechanics to write the equations in a compact form and to emphasise their structure.

3. Summary of principal results. We start by listing our results (for the case of random forcing, where $D(p) \propto 1 /|p|)$. We construct the eigenvalues $\lambda_{n}$ and eigenfunctions $\psi_{n}(z)$ of the operator $\hat{H}$. We identify raising and lowering operators $\hat{A}^{+}$and $\hat{A}$ which map one eigenfunction to another with respectively one more or one fewer nodes. We use these to show that the spectrum of $\hat{H}$ consists of two superposed equally spaced spectra (ladder spectra) for even and odd parity states:

$$
\lambda_{n}^{+}=-3 n, \quad \lambda_{n}^{-}=-(3 n+2), \quad n=0 \ldots \infty .
$$

The spectrum of the Hamiltonian (7) is displayed on the rhs of of Fig. 1. It unusual because the odd-even step is different from the even-odd step, due to the singularity of the Hamiltonian at $z=0$. Our raising and lowering operators allow us to obtain matrix elements required for calculating expectation values. One interesting result is the variance of the momentum for a particle starting at rest at $t=0$ :

$$
\left\langle p^{2}(t)\right\rangle=\left(\frac{p_{0} D_{1}}{\gamma}\right)^{2 / 3} \frac{3^{7 / 6} \Gamma(2 / 3)}{2 \pi}\left(1-\mathrm{e}^{-3 \gamma t}\right)^{2 / 3} .
$$

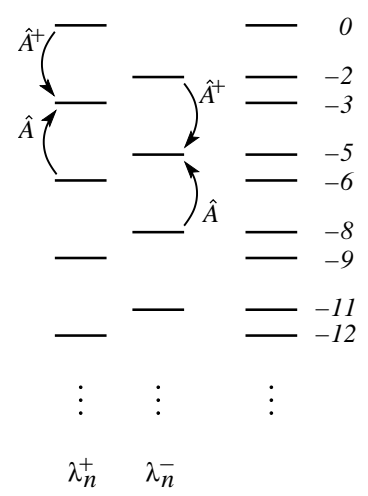

FIG. 1: The spectrum of $\hat{H}$ (right) is the sum of two equally spaced (ladder) spectra $\lambda_{n}^{-}$and $\lambda_{n}^{+}$shifted w.r.t each other (left).

This is reminiscent of equation (11) for the standard Ornstein-Uhlenbeck process, however (9) exhibits anomalous diffusion for small times. At large times $\left\langle p^{2}(t)\right\rangle$ converges to the expectation of $p^{2}$ with the stationary (non-Maxwellian) momentum distribution

$$
P_{0}(p)=\mathcal{N} \exp \left(-\gamma|p|^{3} /\left(p_{0} D_{1}\right)\right)
$$

$(\mathcal{N}$ is a normalisation constant). At large times the dynamics of the spatial displacement is diffusive $\left\langle x^{2}(t)\right\rangle \sim$ $2 \mathcal{D}_{x} t$ with diffusion constant

$$
\mathcal{D}_{x}=\frac{\left(p_{0} D_{1}\right)^{2 / 3}}{m^{2} \gamma^{5 / 3}} \frac{\pi 3^{-5 / 6}}{2 \Gamma(2 / 3)^{2}} F_{32}\left(\frac{1}{3}, \frac{1}{3}, \frac{2}{3} ; \frac{5}{3}, \frac{5}{3} ; 1\right)
$$

(here $F_{32}$ is a hypergeometric function). At small times, by contrast, we obtain anomalous diffusion

$$
\left\langle x^{2}(t)\right\rangle=\mathcal{C}_{x}\left(\left(p_{0} D_{1}\right)^{2 / 3} \gamma^{-8 / 3} m^{-2}\right) t^{8 / 3}
$$

where the constant $\mathcal{C}_{x}$ is given by (30) below.

4. Ladder operators and eigenfunctions. The eigenfunction of the Fokker-Planck equation (5) are alternately even and odd functions, defined on the interval $(-\infty, \infty)$. The operator $\hat{H}$, describing the limiting case of this Fokker-Planck operator, is singular at $z=0$. We identify two eigenfunctions of $\hat{H}$ by inspection, $\psi_{0}^{+}(z)=\mathcal{C}_{0}^{+} \exp \left(-|z|^{3} / 6\right)$ which has eigenvalue $\lambda_{0}^{+}=0$ and $\psi_{0}^{+}(z)=\mathcal{C}_{0}^{-} z|z| \exp \left(-|z|^{3} / 6\right)$ with eigenvalue $\lambda_{0}^{-}=-2$. These eigenfunctions are of even and odd parity, respectively (zero and one node, respectively). Our approach to determining the full spectrum will be to define a raising operator $\hat{A}^{+}$which maps any eigenfunction $\psi_{n}^{ \pm}(z)$ to its successor with the same parity, $\psi_{n+1}^{ \pm}(z)$, having two additional nodes.

We now list definitions of the operators we use: these include the definitions of the raising and lowering operators, $\hat{A}^{+}$and $\hat{A}$, as well as an alternative representation of the Hamiltonian:

$$
\begin{gathered}
\hat{a}^{ \pm}=\left(\partial_{z} \pm z|z| / 2\right) \\
\hat{A}=\hat{a}^{+}|z|^{-1} \hat{a}^{+}, \quad \hat{A}^{+}=\hat{a}^{-}|z|^{-1} \hat{a}^{-} \\
\hat{H}=\hat{a}^{-}|z|^{-1} \hat{a}^{+}, \quad \hat{G}=\hat{a}^{+}|z|^{-1} \hat{a}^{-} .
\end{gathered}
$$



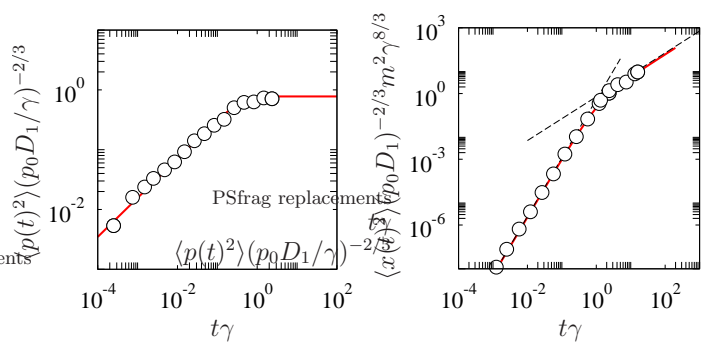

FIG. 2: Shows $\left\langle p^{2}(t)\right\rangle$ and $\left\langle x^{2}(t)\right\rangle$. Computer simulation of the equations of motion $\dot{p}=-\gamma p+f(x, t)$ and $m \dot{x}=p$ (symbols); theory, eqs. (10) and (26), red lines. Also shown are the limiting behaviours for $\left\langle x^{2}(t)\right\rangle$, (11) and (12), at long and short times (dashed lines). In the simulations, $C(X, t)=$ $\sigma^{2} \exp \left[-X^{2} /\left(2 \xi^{2}\right)-t^{2} /\left(2 \tau^{2}\right)\right]$. The parameters were $m=1$, $\gamma=10^{-3}, \xi=0.1, \tau=0.1$, and $\sigma=20$.

Note that $\hat{A}^{+}$is the Hermitian conjugate of $\hat{A}$. The most significant properties of the operators $\hat{A}$ and $\hat{A}^{+}$are

$$
[\hat{H}, \hat{A}]=3 \hat{A} \quad \text { and } \quad\left[\hat{H}, \hat{A}^{+}\right]=-3 \hat{A}^{+}
$$

(the square brackets are commutators). These expressions show that the action of $\hat{A}$ and $\hat{A}^{+}$on any eigenfunction is to produce another eigenfunction with eigenvalue increased or decreased by three, or else to produce a function which is identically zero. The operator $\hat{A}^{+}$adds two nodes, and repeated action of $\hat{A}^{+}$on $\psi_{0}^{+}(z)$ and $\psi_{0}^{-}(z)$ therefore exhausts the set of eigenfunctions. Together with $\lambda_{0}^{+}=0$ and $\lambda_{0}^{-}=-2$ this establishes that the spectrum of $\hat{H}$ is indeed (8). Some other useful properties of the operators of equation (13) are

$$
\left[\hat{A}^{+}, \hat{A}\right]=3(\hat{H}+\hat{G}), \hat{H}-\hat{G}=\hat{I}, \hat{A}^{+} \hat{A}=\hat{H}^{2}+2 \hat{H} .
$$

We represent the eigenfunctions by of $\hat{H}$ by kets $\left|\psi_{n}^{-}\right\rangle$ and $\left|\psi_{n}^{+}\right\rangle$. The actions of $\hat{A}$ and $\hat{A}^{+}$are

$$
\hat{A}^{+}\left|\psi_{n}^{ \pm}\right\rangle=C_{n+1}^{ \pm}\left|\psi_{n+1}^{ \pm}\right\rangle \quad \text { and } \quad \hat{A}\left|\psi_{n}^{ \pm}\right\rangle=C_{n}^{ \pm}\left|\psi_{n-1}^{ \pm}\right\rangle .
$$

where (using (15)) we have $C_{n}^{ \pm}=\sqrt{3 n(3 n \mp 2)}$.

A peculiar feature of $\hat{A}$ and $\hat{A}^{+}$is that they are of second order in $\partial / \partial z$, whereas other examples of raising and lowering operators are of first order in the derivative. The difference is associated with the fact that the spectrum is a staggered ladder: only states of the same parity have equal spacing, so that the raising and lowering operators must preserve the odd-even parity. This suggests replacing a first order operator which increases the quantum number (total number of nodes) by one with a second order operator which increases the quantum number by two, preserving parity.

There is an alternative approach to generating the eigenfunctions of (5). This equation falls into one of the classes considered in [6], and we have written down firstorder operators which map one eigenfunction into another. However, these operators are themselves functions of the quantum number $n$, making the algebra cumbersome. We have not succeeded in reproducing our results with the 'Schrödinger factorisation' method.

5. Propagator and correlation functions. The propagator of the Fokker-Planck equation $\partial_{t^{\prime}} K=\hat{F} K$ can be expressed in terms of the eigenvalues $\lambda_{n}^{\sigma}$ and eigenfunctions $\phi_{n}^{\sigma}(z)=P_{0}^{-1 / 2} \psi_{n}^{\sigma}(z)$ of $\hat{F}$ :

$$
K\left(y, z ; t^{\prime}\right)=\sum_{n=0}^{\infty} \sum_{\sigma= \pm 1} a_{n}^{\sigma}(y) \phi_{n}^{\sigma}(z) \exp \left(\lambda_{n}^{\sigma} t^{\prime}\right) .
$$

Here $y$ is the initial value and $z$ is the final value of the coordinate. The expansion coefficients $a_{n}^{\sigma}(y)$ are determined by the initial condition $K(y, z ; 0)=\delta(z-y)$, namely $a_{n}^{\sigma}(y)=P_{0}^{-1 / 2} \psi_{n}^{\sigma}(y)$. In terms of the eigenfunctions of $\hat{H}$ we have

$$
K\left(y, z ; t^{\prime}\right)=\sum_{n \sigma} P_{0}^{-1 / 2}(y) \psi_{n}^{\sigma}(y) P_{0}^{1 / 2}(z) \psi_{n}^{\sigma}(z) \exp \left(\lambda_{n}^{\sigma} t^{\prime}\right) .
$$

The propagator determines correlation functions. Assuming $z_{0}=0$ we obtain for the expectation value of a function $O(z)$ at time $t$

$$
\left\langle O\left(z\left(t^{\prime}\right)\right)\right\rangle=\sum_{n=0}^{\infty} \frac{\psi_{n}^{+}(0)}{\psi_{0}^{+}(0)}\left\langle\psi_{0}^{+}|O(\hat{z})| \psi_{n}^{+}\right\rangle \exp \left(\lambda_{n}^{+} t^{\prime}\right) .
$$

Similarly, for the correlation function of $O\left(z\left(t_{2}\right)\right)$ and $O\left(z\left(t_{1}\right)\right)\left(\right.$ with $\left.t_{2}^{\prime}>t_{1}^{\prime}>0\right)$

$$
\begin{array}{r}
\left\langle O\left(z\left(t_{2}^{\prime}\right)\right) O\left(z\left(t_{1}^{\prime}\right)\right)\right\rangle=\sum_{n m \sigma} \frac{\psi_{m}^{+}(0)}{\psi_{0}^{+}(0)}\left\langle\psi_{0}^{+}|O(\hat{z})| \psi_{n}^{\sigma}\right\rangle \\
\times\left\langle\psi_{n}^{\sigma}|O(\hat{z})| \psi_{m}^{+}\right\rangle \exp \left[\lambda_{n}^{\sigma}\left(t_{2}^{\prime}-t_{1}^{\prime}\right)+\lambda_{m}^{+} t_{1}^{\prime}\right] .
\end{array}
$$

6. Momentum diffusion. To determine the timedependence of $\left\langle p^{2}(t)\right\rangle$ we need to evaluate the matrix elements $Y_{0 n}=\left\langle\psi_{0}^{+}\left|\hat{z}^{2}\right| \psi_{n}^{+}\right\rangle$. A recursion for these elements is obtained as follows. Let $Y_{0 n+1}=$ $\left\langle\psi_{0}^{+}\left|\hat{z}^{2} \hat{A}^{+}\right| \psi_{n}^{+}\right\rangle / C_{n+1}^{+}$. Write $\hat{z} \hat{A}^{+}=\hat{z} \hat{G}+\hat{z}\left(\hat{A}^{+}-\hat{G}\right)=$ $\hat{z}(\hat{H}-\hat{I})+\hat{z}\left(\hat{A}^{+}-\hat{G}\right)$. It follows

$$
\left\langle\psi_{0}^{+}\left|\hat{z}^{2} \hat{A}^{+}\right| \psi_{n}^{+}\right\rangle=\left(\lambda_{n}^{+}-1\right) Y_{0 n}+\left\langle\psi_{0}^{+}\left|\hat{z}^{2}\left(\hat{A}^{+}-\hat{G}\right)\right| \psi_{n}^{+}\right\rangle .
$$

Using $\left(\hat{A}^{+}-\hat{G}\right)=-\hat{z} \hat{a}^{-}$and $\left[\hat{z}^{3}, \hat{a}^{-}\right]=-3 \hat{z}^{2}$ we obtain $Y_{0 n+1}=\lambda_{n}^{+}+2 Y_{0 n} / C_{n+1}^{+}$, and together with $Y_{00}=$ $3^{7 / 6} \Gamma(2 / 3) /(2 \pi)$ this gives

$$
Y_{0 n}=(-1)^{n+1} \frac{3^{17 / 12} \Gamma(2 / 3)^{3 / 2}}{\sqrt{2} \pi^{3 / 2}(3 n-2)} \frac{\sqrt{\Gamma(n+1 / 3)}}{\sqrt{\Gamma(n+1)}} .
$$

We also find

$$
\psi_{n}^{+}(0) / \psi_{0}^{+}(0)=(-1)^{n} \sqrt{\frac{\sqrt{3} \Gamma(2 / 3)}{2 \pi} \frac{\Gamma(n+1 / 3)}{\Gamma(n+1)}}
$$

and after performing the sum in (19) we return to dimensional variables. The final result is (9). 
7. Spatial diffusion. The time-dependence of $\left\langle x_{t}^{2}\right\rangle$ is determined in a similar fashion, using (20) and (23):

$$
\left\langle x^{2}(t)\right\rangle=\frac{1}{\gamma^{2}}\left(\frac{p_{0} D_{1}}{\gamma}\right)^{2 / 3} \frac{1}{m^{2}} \int_{0}^{t^{\prime}} \mathrm{d} t_{1}^{\prime} \int_{0}^{t^{\prime}} \mathrm{d} t_{2}^{\prime}\left\langle z_{t_{1}^{\prime}} z_{t_{2}^{\prime}}\right\rangle .
$$

The matrix elements $Z_{m n}=\left\langle\psi_{m}^{+}|\hat{z}| \psi_{n}^{-}\right\rangle$are found by a recursion method, analogous to that yielding (22):

$$
\begin{aligned}
Z_{m n} & =(-1)^{m-n} \frac{3^{5 / 6}}{6 \pi}(m+n+1) \Gamma(2 / 3) \\
& \times \frac{\sqrt{\Gamma(n+1) \Gamma(m+1 / 3)}}{\sqrt{\Gamma(m+1) \Gamma(n+5 / 3)}} \frac{\Gamma(n-m+1 / 3)}{\Gamma(n-m+2)} .
\end{aligned}
$$

for $l \geq m-1$ and zero otherwise. Using (20)

$$
\left\langle x^{2}(t)\right\rangle=\frac{\left(p_{0} D_{1}\right)^{2 / 3}}{m^{2} \gamma^{5 / 3}} \sum_{k=0}^{\infty} \sum_{l=k-1}^{\infty} A_{k l} T_{k l}\left(t^{\prime}\right)
$$

with $A_{k l}=\left(\psi_{k}^{+}(0) / \psi_{0}^{+}(0)\right) Z_{0 l} Z_{k l}$ and

$$
\begin{aligned}
T_{k l}\left(t^{\prime}\right)= & \int_{0}^{t^{\prime}} \mathrm{d} t_{1}^{\prime} \int_{t_{1}^{\prime}}^{t^{\prime}} \mathrm{d} t_{2}^{\prime} \mathrm{e}^{\lambda_{l}^{-}\left(t_{2}^{\prime}-t_{1}^{\prime}\right)+\lambda_{k}^{+} t_{1}^{\prime}} \\
& +\int_{0}^{t^{\prime}} \mathrm{d} t_{1}^{\prime} \int_{0}^{t_{1}^{\prime}} \mathrm{d} t_{2}^{\prime} \mathrm{e}^{\lambda_{l}^{-}\left(t_{1}^{\prime}-t_{2}^{\prime}\right)+\lambda_{k}^{+} t_{2}^{\prime}}
\end{aligned}
$$

We remark upon an exact sum-rule for the $A_{k l}$, and also on their asymptotic form for $k \gg 1, l \gg 1$ :

$$
\sum_{k=0}^{l} A_{k l}=0, A_{k l} \sim \frac{\Gamma(2 / 3)^{2}}{3^{1 / 3} 4 \pi^{2}} \frac{k+l}{k^{2 / 3} l^{4 / 3}(l-k)^{5 / 3}} .
$$

We now show how to derive the limiting behaviours (11) and (12), shown as dashed lines in Fig. 2 At large time $x$ evolves diffusively: $\left\langle x^{2}\right\rangle \sim 2 \mathcal{D}_{x} t$, with the diffusion constant obtained from the equilibrium momentum correlation function:

$$
\begin{aligned}
\mathcal{D}_{x} & =\frac{1}{2 m^{2} \gamma}\left(\frac{p_{0} D_{1}}{\gamma}\right)^{2 / 3} \lim _{T \rightarrow \infty} \int_{-\infty}^{\infty} \mathrm{d} t^{\prime}\left\langle z_{T} z_{t^{\prime}+T}\right\rangle \\
& =\frac{-1}{2 m^{2} \gamma}\left(\frac{p_{0} D_{1}}{\gamma}\right)^{2 / 3} \sum_{n=0}^{\infty} \frac{Z_{0 n}^{2}}{\lambda_{n}^{-}}
\end{aligned}
$$

which evaluates to (11). At small values of $t^{\prime}$ the double sum (26) is dominated by the large- $k, l$ terms. We thus evaluate the small- $t^{\prime}$ behaviour by approximating the sums in (26) by integrals and using the asymptotic form for the coefficients $A_{k l}$. There is a non-integrable divergence of $A(k, l)$ as $k \rightarrow l$, which can be cancelled by using the sum rule in equation (28). We obtain the limiting behaviour (12) with

$$
\begin{aligned}
\mathcal{C}_{x} & =-C \int_{0}^{\infty} \mathrm{d} x x^{-8 / 3} \\
& \times \int_{0}^{1} \mathrm{~d} y\left[\frac{a(x)-a(x y)}{1-y}+x a^{\prime}(x)\right] \frac{1+y}{y^{2 / 3}(1-y)^{5 / 3}}
\end{aligned}
$$

where $a(x)=[1-\exp (-x)] / x$ and $C=$ $3^{-7 / 3} \Gamma(2 / 3) /\left(2 \pi^{2}\right)$. The integral is convergent and can be evaluated numerically to give $\mathcal{C}_{x}=0.57 \ldots$ This is in good agreement with a numerical evaluation of the sum (26), as shown in Fig. 2]

8. Gradient-force case. In the case where the force is the gradient of a potential function, we have $D(p)=$ $D_{3} p_{0}^{3} /|p|^{3}+O\left(p^{-4}\right)[7]$. In dimensionless variables the Fokker-Planck equation is

$$
\partial_{t^{\prime}} P=\partial_{z}\left(z P+|z|^{-3} \partial_{z} P\right) \equiv \hat{F} P
$$

instead of (6). This Fokker-Planck equation has the non-Maxwellian equilibrium distribution $P_{0}(z)=$ $\exp \left(-|z|^{5} / 5\right)$. The raising and lowering operators are of the form $\hat{A}^{+}=\hat{a}^{-}|z|^{-3} \hat{a}^{-}$and $\hat{A}=\hat{a}^{+}|z|^{-3} \hat{a}^{+}$ with $\hat{a}^{ \pm}=\left(\partial_{z} \pm z|z|^{3} / 2\right)$. The analogue of (14) is $[\hat{H}, \hat{A}]=5 \hat{A},\left[\hat{H}, \hat{A}^{+}\right]=-5 \hat{A}^{+}$, and the eigenvalues are $0,-4,-5,-9,-10,-14,-15, \ldots$. In this case, too, a closed expression for example for $\left\langle p^{2}(t)\right\rangle$ can be obtained which is analogous to (1), but which exhibits anomalous diffusion:

$$
\begin{aligned}
\left\langle p_{t}^{2}\right\rangle & =\left(\frac{p_{0} D_{3}}{\gamma}\right)^{2 / 5} \frac{5^{2 / 5} \sin (\pi / 5)}{\pi} \\
& \times \Gamma(3 / 5) \Gamma(4 / 5)\left(1-\mathrm{e}^{-5 \gamma t}\right)^{2 / 5}
\end{aligned}
$$

The short-time anomalous diffusion is consistent with the asymptotic scaling obtained in [7, 8] for the special case of undamped stochastic acceleration.

A full account of our results, and their extension to higher dimensions, will be published elsewhere.

9. Acknowledgements. We thank Stellan Östlund for illuminating discussions. MW thanks JSPS for a visiting fellowship.
[1] N. G. van Kampen, Stochastic processes in physics and chemistry, 2nd ed., North-Holland, Amsterdam, (1992).

[2] P. A. Sturrock, Phys. Rev., 141, 186, (1966).

[3] U. Achatz, J. Steinacher and R. Schlickeiser, Astron. Astrophys., 250, 266-79, (1991).

[4] O. Stawicki, J. Geophys. Res., 109, A04105, (2004).

[5] P. A. M. Dirac, The principles of Quantum Mechanics,
Oxford University Press, Oxford, (1930).

[6] L. Infeld and T. E. Hull, Rev. Mod. Phys., 33, 21, (1951).

[7] L. Golubovic, S. Feng, and F.-A. Zeng, Phys. Rev. Lett., 67, 2115, (1991).

[8] M. N. Rosenbluth, Phys. Rev. Lett., 69, 1831, (1992). 